\section{POS0761 INVESTIGATION ON THE EFFECT AND MECHANISM OF ABNORMALLY ACTIVATED CD8+ T CELLS FROM BONE MARROW ON HEMATOPOIETIC STEM CELLS IN PATIENTS WITH SYSTEMIC LUPUS ERYTHEMATOSUS}

T. Fu ${ }^{1}$, Y. Yang ${ }^{1}$, X. Gu' ${ }^{2}$, C. Dong ${ }^{2}$, R. Zhao ${ }^{1}$, J. Ji ${ }^{2}$, Z. Xue ${ }^{1}$, X. Zhang ${ }^{3}$, Z. Gu ${ }^{2}$. ${ }^{1}$ Affiliated Hospital of Nantong University, Research Center of Clinical Medicine, Nantong, China; ${ }^{2}$ Affiliated Hospital of Nantong University, Department of Rheumatology, Nantong, China; ${ }^{3}$ Institute Pasteur of Shanghai Chinese Academy of Sciences, Key Laboratory of Molecular Virology \& Immunology, Shanghai, China

Background: SLE is an autoimmune disease characterized by the abnormal function of lymphocytes. The impairment of hematopoietic function of bone marrow participates in its pathogenesis, in which T cells play an important role. However, study on bone marrow T cells in SLE patients is very limited.

Objectives: This study aims to characterize the phenotype and molecular characteristics of abnormally activated CD8+T cells in bone marrow of SLE patients and explore the mechanism of hematopoietic stem cells (HSCs) reduction caused by the abnormally activated CD8+T cells in bone marrow of patients with SLE.

Methods: A total of 8 SLE patients and 5 age- and sex-matched controls were recruited in our study. Among them, 3 SLE patients and 4 donors were collected bone marrow and peripheral blood samples for Single-cell RNA sequencing (scRNA-seq) and functional studies. BM and peripheral T cell subsets were measured by flow cytometry. Plasma cytokines and secreted immunoglobulins were detected by Luminex. Disease activity of SLE patients was measured using the SLE Disease Activity Index (SLEDAI). All analyses were performed using R language and Flowjo 9

Results: In the present study, SLE patients had increased CD8+T\% $\alpha \beta T$ cells and decreased CD4+T\% $\alpha \beta T$ cells in bone marrow of SLE, compared to healthy controls. A large number of CD38+HLADR+CD8+T cells existed in the bone marrow and peripheral blood of SLE patients. Those patients also showed reduced number of HSCs, and with a downward trend of the numbers of peripheral red blood cells, white blood cells, neutrophils, hemoglobin, and platelets. By scRNA-seq, the CD38+HLADR+CD8+T cells contained high levels of GZMK, GZMA, PRF1, IFNG, and TNF in the bone marrow of SLE patients. the CD38+HLADR+CD8+T cells exhibited significant relationship with HSCs, white blood cells, neutrophils, and platelets. Conclusion: These findings demonstrated that the abnormally activated CD8+T cells in bone marrow can reduce the number of HSCs by the expression of killer molecules, which contributes to the impairment of hematopoietic function and the development of SLE. This project focuses on the specific bone marrow $T$ cell subset in SLE. The completement of this project provides information for exploring the mechanism of hematopoiesis involvement.

\section{REFERENCES:}

[1] Anderson E, Shah B, Davidson A, Furie R. Lessons learned from bone marrow failure in systemic lupus erythematosus: Case reports and review of the literature. Semin Arthritis Rheum. 2018:48(1):90-104

[2] Sun LY, Zhou KX, Feng XB, Zhang HY, Ding XQ, Jin O, Lu LW, Lau CS, Hou YY, Fan LM. Abnormal surface markers expression on bone marrow CD34+cells and correlation with disease activity in patients with systemic lupus erythematosus. Clin Rheumatol. 2007;26(12):2073-2079.

Acknowledgements: We want to thank Lu Meng, Teng Li, Wei Zhou, and Jiaxin Guo for their assistance with this study.

Disclosure of Interests: None declared

DOI: 10.1136/annrheumdis-2021-eular.3060

\section{POS0762 \\ CAN THE SLE-DAS SUBSTITUTE BILAG TO MEASURE LUPUS DISEASE ACTIVITY IN CLINICAL TRIALS? POST-HOC ANALYSIS OF THE BLISS-76 TRIAL}

D. Jesus ${ }^{1,2}$, A. Matos ${ }^{3,4}$, C. Henriques ${ }^{3,5}$, A. Doria ${ }^{6}$, L. Inês ${ }^{2,7} .{ }^{1}$ Centro Hospitalar de Leiria, Rheumatology Department, Leiria, Portugal; ${ }^{2}$ University of Beira Interior, Faculty of Health Sciences, Covilhã, Portugal: ${ }^{3}$ Institute of Viseu, Technology and Management, Viseu, Portugal; ${ }^{4}$ Research Centre in Digital Services, C/SeD, Viseu, Portugal; ${ }^{5}$ University of Coimbra, Centre for Mathematics, Coimbra, Portugal; ${ }^{6}$ University of Padova, Division of Rheumatology, Padova, Italy; ${ }^{7}$ Centro Hospitalar e Universitário de Coimbra, CHUC Lupus Clinic, Rheumatology Department, Coimbra, Portugal

Background: The primary endpoint for randomized clinical trials (RCT) in Systemic lupus erythematosus (SLE) is usually defined as proportion of responders in a composite index. The most widely used are British Isles Lupus Assessment Group (BILAG)-based Composite Lupus Assessment (BICLA) and Systemic Lupus Responder Index (SRI). Both comprise BILAG along with SLE Disease Activity Index (SLEDAI). BICLA and SRI are complex and time-consuming to assess. The SLE Disease Activity Score (SLE-DAS) is an easy to apply, validated, continuous disease activity measure, highly correlated with SLEDAI, with higher accuracy and sensitivity-to-change as compared to SLEDAI ${ }^{1}$.
We hypothesize that SLE-DAS can also identify the SLE disease activity information from BILAG, thus dispensing the use of composite indexes for RCT. Objectives: To compare the ability of the SLE-DAS and the Safety of Estrogen in Lupus National Assessment (SELENA)-SLEDAl to discriminate between BILAG classification of mild vs. moderate vs. severe disease activity.

Methods: Post-hoc analysis of all intention-to-treat patients in the BLISS-76 (NCT00410384) RCT at the baseline study visit. SELENA-SLEDAI and BILAG were assessed at time of the study visits and SLE-DAS was retrospectively scored from the study database. Patients' disease activity was classified as: (i) mild (no BILAG B or A scores in any organ domain); (ii) moderate (1 BILAG B, no A scores); (iii) severe ( $\geq 2$ BILAG B and/or $\geq 1$ BILAG A). Ability of the SLE-DAS and SELENA-SLEDAI to differentiate between: (i) mild vs. moderate/severe disease activity; (ii) mild/moderate vs. severe disease activity (according to BILAG), were evaluated using receiver operating characteristic (ROC) analysis. The area under the ROC curves (AUCs) with $95 \%$ confidence intervals $(95 \% \mathrm{Cl}$ ) as a measure of discriminatory ability of the SLE-DAS and SELENA-SLEDAI were compared using Delong's test for two correlated curves. Because AUC measurements might have restricted accuracy for imbalanced datasets, precision-recal (PR) curves and area under PR curves (AUC-PR) were also performed. Statistical significance was set at 0.05 .

Results: We included 819 patients, classified by BILAG as presenting mild $(7.7 \%)$, moderate $(28.8 \%)$ or severe $(63.5 \%)$ disease activity. To differentiate mild vs. moderate/severe disease activity, the discriminatory ability of SLE-DAS was outstanding (AUC $0.948 ; 95 \% \mathrm{Cl} 0.923-0.973$ ), while that of SELENA-SLEDAI was acceptable (AUC $0.729 ; 95 \% \mathrm{Cl} 0.657-0.801)(p<0.005)$ (figure $1 \mathrm{~A})$. To differentiate mild/moderate vs. severe disease activity, the discriminatory ability of SLE-DAS was excellent (AUC $0.873 ; 95 \% \mathrm{Cl} 9.846-0.899$ ), while that of SELENA-SLEDAI was acceptable (AUC $0.707 ; 95 \% \mathrm{Cl} 0.670-0.744)(\mathrm{p}<0.005)$ (figure 1B). The AUC-PR confirmed the higher performance of SLE-DAS over SELENA-SLEDAI to differentiate mild vs. moderate/severe disease activity ( 0.995 vs. 0.965 , respectively) (figure $1 \mathrm{C}$ ) and mild/moderate vs. severe disease activity (0.902 vs. 0.794 , respectively) (figure 1D).
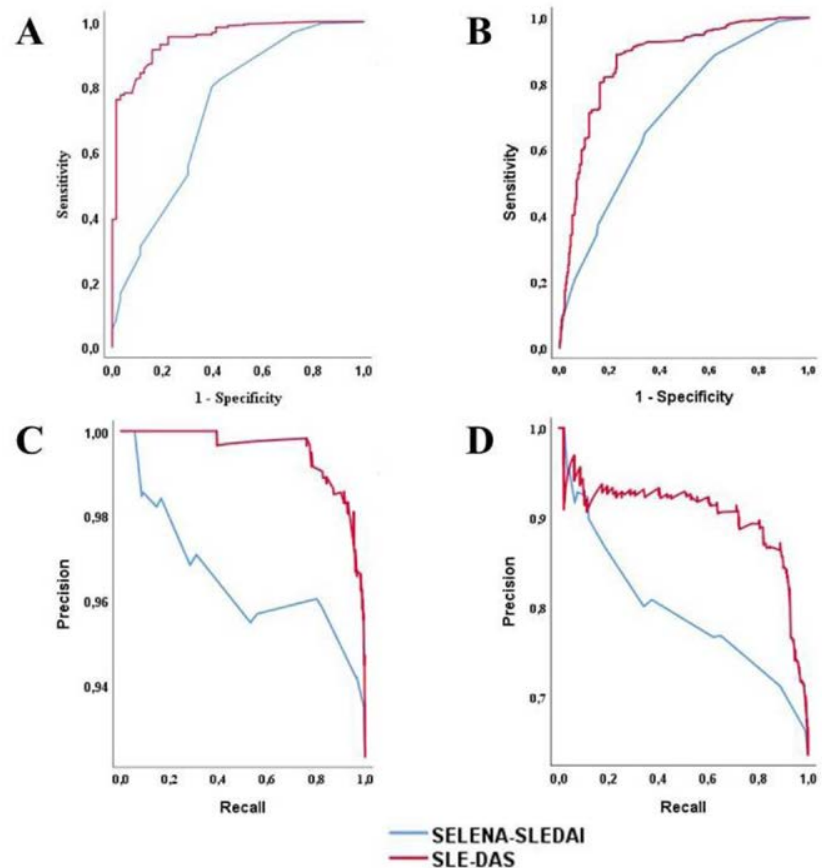

Figure 1. Receiver operating characteristics (ROC) curves comparing the ability of the SLEDAS and SELENA-SLEDAI to differentiate (A) mild $v s$. moderate/severe disease activity and (B) mild/moderate vs. severe disease activity, as assessed by BILAG; and Precision-recall (PR) curves comparing the performance of the SLE-DAS and SELENA-SLEDAI to differentiate (C) mild vs. moderate/severe disease activity and (D) mild/moderate vs. severe disease activity.

Conclusion: The SLE-DAS presents excellent performance in assessing SLE disease activity categorized by BILAG scores, which is not the case for SELENA-SLEDAI. Further studies will aim to better define ability of SLE-DAS to substitute composite responder indices.

\section{REFERENCES:}

[1] Jesus D, et al. Derivation and validation of the SLE Disease Activity Score

(SLE-DAS): a new SLE continuous measure with high sensitivity for changes in disease activity. Ann Rheum Dis 2019;78:365-71.

Acknowledgements: The authors would like to thank GlaxoSmithKline (Uxbridge, UK) for granting access to the data from the BLISS-76 trial through the Clinical Study Data Request consortium. 
Disclosure of Interests: None declared

DOI: 10.1136/annrheumdis-2021-eular.3162

\section{POS0763 PERFORMANCE OF THE NEW ACR/EULAR 2019 CLASSIFICATION CRITERIA FOR SYSTEMIC LUPUS ERYTHEMATOSUS (SLE) IN A COHORT OF ARGENTINIAN PATIENTS}

J. M. Dapeña ${ }^{1}$, E. R. Serrano ${ }^{1}$, J. M. Bande ${ }^{1}$, M. A. Medina ${ }^{1}$, D. S. Klajn ${ }^{1}$, J. A. Caracciolo ${ }^{1}$, C. Castro ${ }^{2}$, J. Morbiducci ${ }^{2}$, A. L. Mercé2, R. Tralice ${ }^{1,2}$, G. V. Espasa ${ }^{3}$, S. Yessika Jackeline ${ }^{3}$, M. L. Leguizamón ${ }^{3}$, M. A. Pera ${ }^{3}$, V. I. Bellomio ${ }^{3}$, M. S. Yacuzzi ${ }^{4}$, M. Machado Escobar ${ }^{4}$, M. Cosentino ${ }^{5}$, L. Garcia ${ }^{5}$, M. García ${ }^{5}$, C. Aeschlimann ${ }^{6}$, G. Gomez ${ }^{7}$, N. Perez ${ }^{7}$, S. Papasidero ${ }^{1}{ }^{1}$ Hospital General de Agudos Dr. Enrique Tornú, Servicio de Reumatología, Buenos Aires, Argentina; ${ }^{2}$ Hospital General de Agudos Bernardino Rivadavia, Servicio de Reumatología, Buenos Aires, Argentina; ${ }^{3}$ Hospital Ángel C. Padilla Juan Bautista Alberdi, Servicio de Reumatología, San Miguel de Tucumán, Argentina; ${ }^{4}$ Hospital Eva Perón de Tucumán, Servicio de Reumatología, Banda del Río Sali, Argentina; ${ }^{5}$ H.I.G.A. San Martín de La Plata, Servicio de Reumatología, La Plata, Argentina; ${ }^{6}$ Hospital Provincial de Rosario, Servicio de Reumatología, BZL, Argentina; ${ }^{7}$ Instituto de Investigaciones Médicas Alfredo Lanari, Servicio de Reumatología, Buenos Aires, Argentina

Background: In 2019 ACR and EULAR published in joint collaboration the new classification criteria for Systemic Lupus Erythematosus (SLE). Compared to the previous ones, these criteria have shown higher sensitivity and specificity in multiple cohorts. To our knowledge, its performance has not been evaluated in a cohort of patients with rheumatological diseases living in Argentina.

Objectives: The aim of this study was to evaluate the sensitivity and specificity of the ACR/ EULAR 2019 criteria in a cohort of patients with connective tissue diseases residing in Argentina. Secondary objectives were to determine the Likelihood Ratio (LR) of these criteria and the correlation of their global score with activity and damage indexes of the disease.

Methods: Multicentre, retrospective and analytical study. Patients $\geq 18$ years old with diagnosis of SLE (ACR 1997/SLICC 2012) without other associated collagen diseases (case group), and patients with other non-SLE connective tissue diseases (control group) were included. Those with active infectious disease, oncohematological disease, drug-induced lupus and overlap syndrome were excluded. Sociodemographic data, characteristics of the disease and treatment were recorded. In addition, activity and damage indexes were recorded in the group with SLE.

Three SLE experts, blinded to the diagnosis determined, for every individual if the patient had SLE or another rheumatological disease. An interrater agreement of $100 \%$ (including the 3 evaluators) was considered "defined SLE" and used as gold standard. In all cases, ACR 1997/SLICC 2012/ACR / EULAR 2019 criteria were applied and compared with the gold standard. Statistical analysis: Descriptive statistics was estimated. Sensitivity, specificity, positive and negative LR of the criteria were determined. The association between the final score of the ACR-EULAR 2019 criteria and the disease activity and damage indexes were estimated with Spearman correlation test. STATA 15.0 was used for data analysis.

Results: A total of 365 patients from 7 centres in Argentina were included. A One hundred and eighty-three belonged to the SLE group: $92.3 \%$ women, mean age 39 years (SD 13.3), median disease duration 92 months (IQR 37-150). The most frequent manifestations of the disease were mucocutaneous (94\%), musculoskeletal $(82.5 \%)$ and haematological $(69 \%)$. All patients presented ANA +, $88 \%$ hypocomplementemia, $69.4 \%$ Anti-DNA and $19.5 \%$ antiphospholipid antibodies. Median SLEDAI and SLICC were 2 (IQR 0-6) and 0 (IQR 0-1), respectively. In the control group, 182 patients were recruited: $84 \%$ women, mean age 53.6 years (SD 14.2) and median disease duration 82.5 months (IQR 38-151). The most frequent diseases were Rheumatoid Arthritis (46.1\%), Scleroderma (18.1\%) and Sjögren's Syndrome (16.5\%) and most common manifestations were musculoskeletal (81.9\%), immunological $(73.6 \%)$ and constitutional (25.3\%). A total of $62.6 \%$ of patients presented ANA+, $8.6 \%$ hypocomplementemia, and $1.3 \%$ Antiphospholipid antibodies.

Ninety-one percent of patients in the case group were classified as defined SLE and $3.8 \%$ in the control group.

The ACR / EULAR 2019 Criteria showed a 99.4\% sensitivity and an $89.1 \%$ specificity, with a LR+ of 9.1 and a LR- of 0.007 . The sensitivity and specificity of SLICC 2012 criteria were $98.3 \%$ and $88 \%$, respectively with a LR+ of 8.2 and a LR- of 0.02 ; and the ACR 1997 criteria showed a $93.96 \%$ sensitivity and $90.1 \%$ specificity, with $L R+$ of 8.21 and $L R$ - of 0.07 .

The correlations between the ACR/EULAR 2019 Criteria global score, and activity and damage indexes were 0.19 and -0.006 , respectively.

Conclusion: The new ACR / EULAR 2019 criteria have shown high sensitivity, a specificity comparable to its predecessors, and a higher ability to distinguish SLE from other diseases and to exclude it in non-SLE patients. No correlation was observed between the criteria scores and activity and damage indexes.

\section{REFERENCES:}

[1] Aringer M, Costenbader K, Daikh D, et al 2019 EULAR/ACR classification criteria for SLE. Ann Rheum 2019; 78: 1151-1159.

Disclosure of Interests: None declared

DOI: 10.1136/annrheumdis-2021-eular.3172

\section{POS0764 EULAR RECOMMENDATION-BASED QUALITY INDICATORS (QIS) FOR SYSTEMIC LUPUS ERYTHEMATOSUS (SLE): ELABORATION, FINAL SET, PERFORMANCE AND INITIAL VALIDATION}

K. Chavatza ${ }^{1}$, M. Kostopoulou' ${ }^{2}$, D. Nikolopoulos ${ }^{1}$, O. Gioti ${ }^{3}$, K. Togia $^{1}$ S. Flouda', N. Kapsala', M. Kosmetatou', G. S. Moysidou', A. Grivas ${ }^{1}$ A. Pieta ${ }^{1}$, A. Ntourou ${ }^{1}$, P. Rapsomaniki ${ }^{1}$, T. Gerogianni ${ }^{1}$, D. Tseronis ${ }^{1}$, M. Aggelakos ${ }^{1}$, T. Karageorgas ${ }^{1}$, P. Katsimpri ${ }^{1}$, L. Andreoli ${ }^{4,5}$, M. Aringer $^{6}$, J. N. Boletis ${ }^{7}$, A. Doria ${ }^{8}$, F. Houssiau ${ }^{9}$, D. Jayne ${ }^{10}$, M. Mosca ${ }^{11}$, E. Svenungsson ${ }^{12}$, A. Tincani ${ }^{13}$, G. Bertsias ${ }^{14}$, A. Fanouriakis ${ }^{1,3}$, D. Boumpas ${ }^{1,15}$. "Attikon" University Hospital of Athens, Rheumatology and Clinical Immunology, Medical School, National and Kapodistrian University of Athens, Athens, Greece; " $\mathrm{G}$. Gennimatas" General Hospital, Department of Nephrology, Athens, Greece; 3"Asklepieion" General Hospital, Voula, Department of Rheumatology, Athens, Greece: ${ }^{4}$ University of Brescia, Department of Clinical and Experimental Sciences, Brescia, Italy; ${ }^{5}$ Spedali Civili, Unit of Rheumatology and Clinical Immunology, Brescia, Italy; ${ }^{6}$ University Medical Center \& Faculty of Medicine Carl Gustav Carus, Technical University of Dresden, Division of Rheumatology, Department of Medicine III, Dresden, Germany; "“Laikon” Hospital, National and Kapodistrian University of Athens, Medical School, Nephrology Department and Renal Transplantation Unit, Athens, Greece; ${ }^{8}$ University of Padova, Rheumatology Unit, Department of Medicine, Padova, Italy; ${ }^{9}$ Université catholique de Louvain, Cliniques Universitaires Saint-Luc, Brussels, Belgium; ${ }^{10}$ Addenbrooke's Hospital, Department of Medicine, Cambridge, United Kingdom; ${ }^{11}$ University of Pisa, Rheumatology Unit, Department of Clinical and Experimental Medicine, Pisa, Italy: ${ }^{12}$ Karolinska Institutet and Karolinska University Hospital, Department of Medicine, Rheumatology Unit, Stockholm, Sweden; ${ }^{13}$ University of Brescia, Rheumatology and Clinical Immunology, Brescia, Italy; ${ }^{14}$ University Hospital of Heraklion, Rheumatology, Clinical Immunology and Allergy, Heraklion, Greece; ${ }^{15}$ Biomedical Research Foundation of the Academy of Athens, Laboratory of Autoimmunity and Inflammation, Athens, Greece

Background: Targets of therapy and quality of care are receiving increased attention in systemic lupus erythematosus (SLE).

Objectives: To develop Quality Indicators (QIs) for the care of SLE patients based on the EULAR recommendations, and assess their performance.

Methods: Using the published EULAR recommendations for SLE, we developed 44 candidate QIs. These were independently rated for validity and feasibility by 12 experts, analysed by a modified RAND/UCLA model and further scrutinized based on the scorings and expert opinion. (Fig.1) Adherence to the final set of Qls was tested in a cohort of 220 SLE patients combined with an assessment on its impact on disease outcomes such as flares, hospitalizations and organ damage.

Results: The panel rated 18 QIs as valid and feasible. These involve diagnosis; disease and damage assessment; monitoring for lupus nephritis and drug toxicity; therapy and targets of therapy; fertility and pregnancy; and adjunct therapy (preventive measures for osteoporosis, vaccination, cardiovascular disease). On average, SLE patients received $54 \%(95 \% \mathrm{Cl} 52-56 \%)$ of the indicated care with adherence ranging from $41 \%$ for Qls related to monitoring to $88 \%$ for treatment-related QIs. Regarding targets of therapy, sustained remission or low disease activity were achieved in $27 \%$, while $94 \%$ of patients received low-dose glucocorticoids, and $92 \%$ the recommended hydroxychloroquine dose. Dependent upon individual QI tested, adherence for lupus nephritis-related Qls was $88 \%$ for receiving appropriate adjunct therapy (ACE inhibitors) to $100 \%$ for being treated with the indicated immunosuppressive treatment. In contrast, adherence to Qls related to preventive measures and other adjunct therapies was moderate to low. Notably, patients who were eligible for cardiovascular risk modification, vaccination, and osteoporosis management received lower quality of care ( $40.5 \%, 47.7 \%$ and $45.5 \%$ respectively) while $91.4 \%$ had sunscreen protection In reference to laboratory work-up and monitoring, complete laboratory work-up at diagnosis was performed in $48 \%$, while disease activity and damage, were fully assessed only in $14.1 \%$ (in three consecutive visits) and $28.6 \%$ (annually) respectively, Similarly, reproductive health and pregnancy counselling adherence rates were modest estimated at $50 \%$ and $62 \%$ respectively. Higher adherence to the indicated care during follow-up (monitoring QIs) was associated with reduced risk for adverse outcomes during the last year of observation (OR $0.97,95 \% \mathrm{Cl}$ 0.96-0.99). Patients who achieved sustained remission or LLDAS, exhibited fewer flares $(O R=0.15$, $p$-value $<0.001)$ and damage accrual $(O R=0.35$, $p$-value $<0.001)$ Of interest, patients who received low-dose of GCs or were appropriately vaccinated, had a lower risk of experiencing a flare ( $\mathrm{OR}=0.23$ and 0.46 respectively). 